\title{
The North Middle Lobe of Centaurus A
}

\author{
Susan G. Neff ${ }^{1}$, Jean A. Eilek ${ }^{2}$ and Frazer N. Owen ${ }^{3}$ \\ ${ }^{1}$ Observational Cosmology Laboratory, NASA's Goddard Space Flight Center, \\ Greenbelt, MD 20771, USA \\ email: susan.g.neff@nasa.gov \\ ${ }^{2}$ Dept. of Physics, New Mexico Tech, and \\ National Radio Astronomy Observatory, Socorro, NM 87801, USA \\ email: jeilek@aoc.nrao.edu \\ ${ }^{3}$ National Radio Astronomy Observatory, Socorro, NM 87801, USA \\ email: fowen@aoc.nrao.edu
}

\begin{abstract}
We present new, deep VLA $327 \mathrm{MHz}$, GALEX Far-UV, and $\mathrm{H} \alpha$ images of the inner $\sim 50 \mathrm{kpc}$ of Centaurus A. We find the structure identified by Morganti et al. 1999 as a possible "large scale jet" is part of a knotty, linear feature within a broader region of diffuse radio emission. The linear feature is coincident with a narrow ribbon of Far-UV and $\mathrm{H} \alpha$ emission that extends 6-35 kpc from the galaxy core, as well with a similar ridge of soft X-ray emission. The Far-UV image also shows that a strong starburst is occurring in the central dusty disk, with a star-formation rate of $\sim 2 \mathrm{M}_{\odot} \mathrm{yr}^{-1}$. We suggest that the various peculiar phenomena seen to the NE of the galaxy can be explained by a wind from the starburst disk, enhanced by energy input from the AGN.
\end{abstract}

Keywords. galaxies: active, galaxies: individual (Cen A), galaxies: jets, galaxies: starburst

Centaurus A / NGC5128 is the nearest active galaxy $(3.8 \mathrm{Mpc})$. It is an excellent laboratory to study how an active galactic nucleus (AGN) interacts with its host galaxy, and with the larger environment. In Cen $\mathrm{A}$, it is not clear how the $5 \mathrm{kpc}$ inner radio jet connects to the $270 \mathrm{kpc}$ outer lobes, what energizes emission line clouds 10-15 kpc from the galaxy center, or what causes asymmetry in radio emission on the $\sim 50 \mathrm{kpc}$ scale.

Our new VLA $90 \mathrm{~cm}$ image (Fig. 1, left) shows diffuse emission extending $\sim 50 \mathrm{kpc}$ NE of the galaxy, but no comparable emission to the SW. There is no indication of a collimated jet connecting the inner radio lobes $(<10 \mathrm{kpc})$ to the outer lobes $(>100 \mathrm{kpc})$, leaving open the question of how the outer lobes, with short radiative lifetimes $(<30$ Myr), are energized. Fig. 1 (middle) and 1 (right) show closeup images of the North Middle Lobe at 90 and $20 \mathrm{~cm}$. The linear radio feature, previously suggested to be a possible "large-scale-jet" (Morganti et al. 1999) is seen to be part of a longer radio feature within the diffuse emission. Three overpressured radio knots are embedded in the linear radio structure; similarly overpressured X-ray knots (Kraft et al. 2009) occur between the radio knots.

Fig. 2 (left) shows the GALEX FUV image of Cen A, and closeup images of the FUV (center) emission compared with a matched deep $\mathrm{H} \alpha$ image (right). FUV and $\mathrm{H} \alpha$ emission extend at least $35 \mathrm{kpc}$ from the core, in what appears to be a twisted ribbon; the FAR-UV / $\mathrm{H} \alpha$ coincidence suggests ongoing star formation along the ribbon.

The FUV also shows a strong central starburst (Fig. 2 left) in the dust disk, which has a star-formation rate of $\sim 2 \mathrm{M}_{\odot} / \mathrm{yr}$, and an age $>50 \mathrm{Myr}$. The starburst is expected to produce a galactic wind, which will interact with the inner radio jet $(<6 \mathrm{kpc})$ and be channeled by earlier radio outflows. The wind is expected to encounter clouds or streams of cold gas remaining from earlier mergers (Schiminovich et al. 1994), as well 

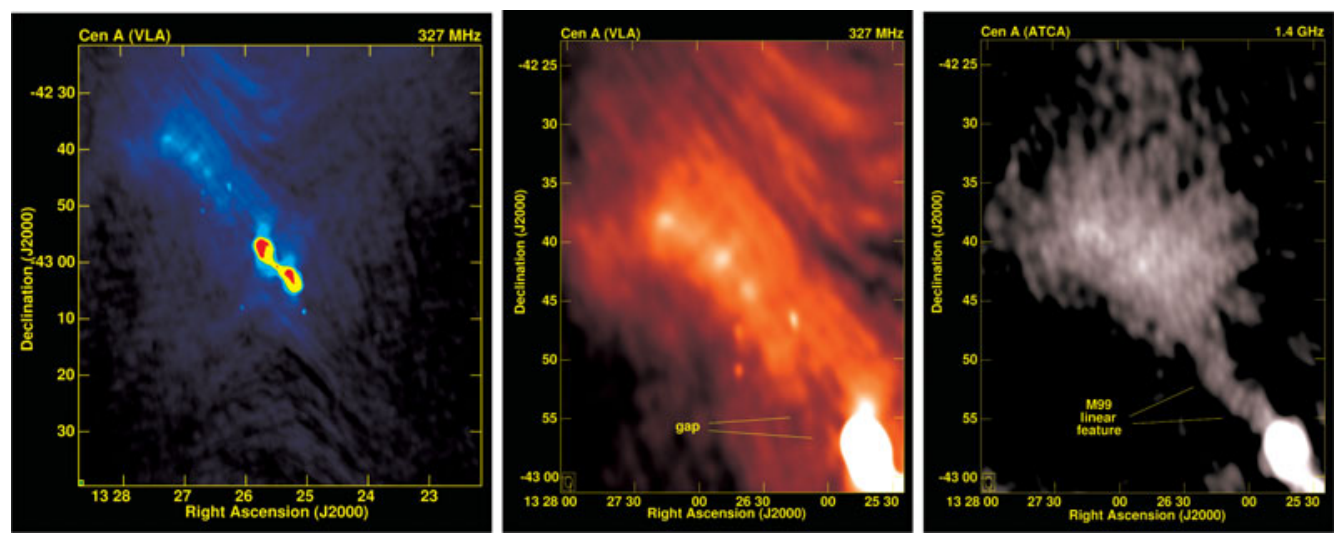

Figure 1. (left) VLA $90 \mathrm{~cm}$ image of Cen A, with radio emission extending $50 \mathrm{kpc}$ NE of the galaxy; (center) VLA $90 \mathrm{~cm}$ image of the North Midde Lobe with three radio knots embedded in a more diffuse structure; (right) $20 \mathrm{~cm}$ image of the same region, from Morganti et al. (1999).
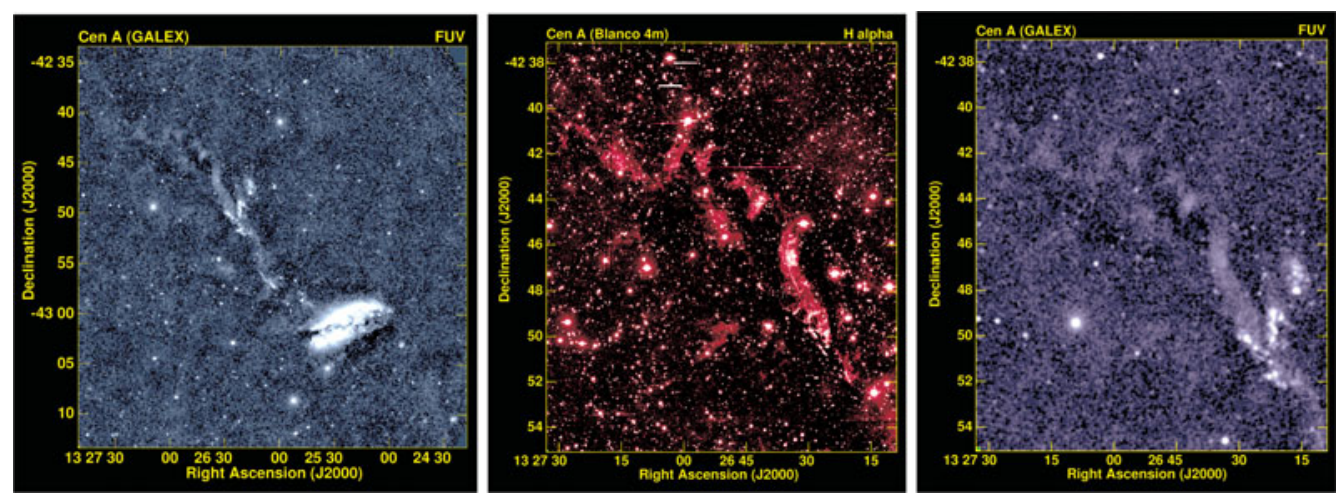

Figure 2. (left) GALEX FUV $\left(\lambda_{e f f}=1539 \AA\right)$ image of Cen A, showing the bright starburst in the galaxy, and the stream of FUV emission extending at least $35 \mathrm{kpc}$ (in projection) NE of the galaxy; close-up views of FUV (center) and $\mathrm{H} \alpha$ (right) images of the "weather" in the North Middle Radio Lobe.

as relativistic plasma remaining from earlier radio outflows. Resulting shocks and turbulence will energize the entire region, as seen in the observed "weather": enhanced radio brightness, overpressured radio and X-ray emitting knots, shock-heated gas filaments, and stimulated star formation $10 \mathrm{~s}$ of $\mathrm{kpc}$ from the galaxy center. The lack of weather to the SW of the galaxy suggests that cold clouds to the SW must lie outside the wind's path. (Further details may be found in Neff et al. 2014 and 2015.)

\section{References}

Kraft, R. P., Forman, W. R., Hardcastle, M. J., et al. 2009, ApJ, 698, 2036

Morganti, R., Kileen, N. E. B., Ekers, R. D., \& Oosterloo, T. A. 1999, MNRAS, 307, 750

Neff, S. G., Eilek, J. A., \& Owen, F. N. 2014, ApJ, submitted

Neff, S. G., Eilek, J. A., \& Owen, F. N. 2015, ApJ, submitted

Schiminovich, D., van Gorkum, J. H., van der Hulst, J. M., \& Kasow, S. 1994, ApJ, 423, L101 